\title{
Size determination of field-induced water menisci in noncontact atomic force microscopy
}

\author{
Montserrat Calleja, Marta Tello, and Ricardo García ${ }^{a}$ \\ Instituto de Microelectrónica de Madrid, CSIC, Isaac Newton 8, 28760 Tres Cantos, Madrid, Spain
}

(Received 25 February 2002; accepted 30 July 2002)

\begin{abstract}
We have studied the dimensions of water capillaries formed by an applied electrical field between an atomic force microscope tip and a flat silicon surface. The lateral and vertical dimensions of the liquid meniscus are in the 5-30 $\mathrm{nm}$ range. The size depends on the duration and strength of the voltage pulse. It increases by increasing the voltage strength or the pulse duration. The meniscus size is deduced from the experimental measurement of the snap-off separation. These results are of special relevance to optimize local oxidation nanolithography. (C) 2002 American Institute of Physics. [DOI: 10.1063/1.1510171]
\end{abstract}

\section{INTRODUCTION}

Local oxidation of semiconductor and metallic surfaces by atomic force microscopy (AFM) is a reliable and versatile method for a variety of nanotechnology applications. ${ }^{1-6} \mathrm{~A}$ key feature of local oxidation lithography is the formation of a liquid meniscus bridging tip and sample surface. ${ }^{7-10}$ Capillary condensation within nanopores is also relevant for other areas such as tribology, ${ }^{11}$ wetting at structured surfaces $^{12}$ and other scanning probe microscopy applications. ${ }^{13}$ Recently several studies have described different aspects of nanometer-size capillaries ${ }^{14-17}$ and electrowetting phenomena. ${ }^{18}$ However, to the best of our knowledge only a few experimental studies have been devoted to nanometer-size menisci in general ${ }^{19-22}$ and nanometer-size capillary-condensed water bridges ${ }^{22-25}$ in particular.

Previously, we have shown that the application of a voltage between tip and surface induces the formation of a water meniscus. ${ }^{7}$ This may happen at separations larger than those predicted by the Kelvin equation. The size of the liquid bridge is critical in local oxidation lithography, because it defines the size of the oxide motives. ${ }^{7}$

In the present work, we study the influence of applied voltage and pulse duration on the dimensions of the water bridges. We also propose a method to determine the meniscus dimensions from snap-off separation measurements. Finally, we study the effect of meniscus dimensions in the final size of oxide marks obtained by local oxidation nanolithography experiments.

\section{MODEL AND EXPERIMENTAL METHODS}

The experiments were performed with an atomic force microscope operated in noncontact mode with additional circuits to apply a voltage between tip and sample. In order to control relative humidity the microscope was placed into a closed box with inlets for dry and $\mathrm{H}_{2} \mathrm{O}$ saturated nitrogen. Doped $n^{+}$-type silicon cantilevers were used (Nanosensors, Germany). The average force constant $\left(k_{c}\right)$ and resonance

a) author to whom correspondence should be addressed; electronic mail: rgarcia@imm.cnm.csic.es frequency $\left(f_{0}\right)$ were about $49 \mathrm{~N} / \mathrm{m}$ and $368 \mathrm{kHz}$, respectively. The cantilever was excited at its resonance frequency. The samples were $p$-type $\mathrm{Si}(100)$ with a resistivity of 14 $\Omega \mathrm{cm}$. Due to exposure to air, the surface has a native oxide layer of about $2 \mathrm{~nm}$. The position of the sample with respect to the tip is derived from measurements of the oscillation amplitude and deflection of the cantilever. The signal from the photodiode is acquired by an oscilloscope to monitor the cantilever movement in real time. Further description of the dynamic parameters needed to operate the AFM in a noncontact mode and to measure tip-sample separation can be found elsewhere. ${ }^{6,7}$

The protocol to form field-induced water bridges with an AFM requires several steps. First the relative humidity, RH, is stabilized at a fixed value in the range $40 \%-50 \%$, so a few monolayers of water may be adsorbed on tip and sample surfaces. $^{26,27}$ The force microscope tip is oscillated at a fixed distance of a few nanometers above the sample surface and a voltage pulse is applied between tip (grounded) and sample. The applied voltage induces the formation of a water bridge between tip and sample whenever the voltage strength is above a certain threshold voltage. ${ }^{7}$ During the application of the voltage pulse the electrostatic force induces the deflection of the cantilever. It also reduces the oscillation amplitude [see A in Fig. 1(c)], however, the tip never gets in contact with the surface. After turning off the voltage, if a water bridge has been condensed between tip and sample, a capillary force damps the oscillation of the cantilever [see B in Fig. 1(c)]. To determine the snap-off separation, the tip and the surface are separated while the tip oscillation is monitored by an oscilloscope. At the snap-off separation there is a sudden increase of the oscillation amplitude. The water capillary is no longer connecting tip and sample. As a consequence, the capillary force damping the cantilever oscillation disappears. When the meniscus evaporates, the tip suddenly recovers its initial oscillation amplitude. The separation at which the liquid bridge is unstable is called the snap-off separation.

We have developed a model to calculate the meniscus dimensions from the snap-off separation. In this model, the profile of the liquid-vapor interface is approximated by an 


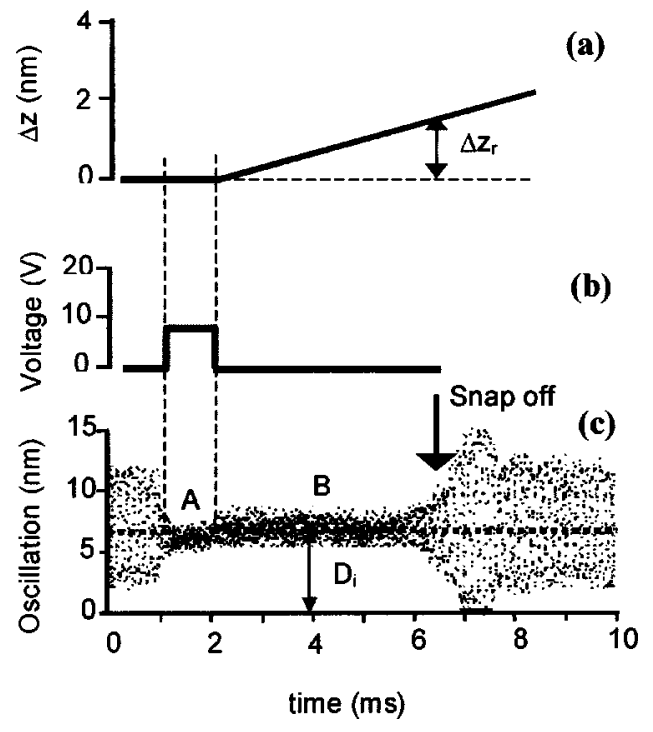

FIG. 1. Measurement of the snap-off separation for a meniscus formed by a pulse of $9 \mathrm{~V}$ and $1 \mathrm{~ms}$. (a) Piezo displacement, $\Delta z$, vs time. (b) Applied voltage. (c) Tip oscillation. $D_{i}=Z_{i}$ represents the initial tip-sample equilibrium distance. Snap-off separation is measured as $D_{\text {snap-off }}=Z_{i}+\Delta z_{\text {snap-off }}$. The zero position in the $y$ axis represents the position of the surface. Relative humidity, $\mathrm{RH}=55 \%$.

arc of a circle. This approximation is considered to be accurate whenever the filling angle $\phi$ and the wetting angle $\theta_{1}$ are both small. ${ }^{28}$ This applies for the present tip-surface geometry where the tip radius is larger than the tip-surface separation. Additionally, both surfaces are hydrophilic, i.e, $\theta_{1} \sim 0^{\circ}$. The model also assumes that each meniscus evolves by keeping constant mean curvature.

From Fig. 2 we derive the following geometric relationships:

$$
\begin{aligned}
& \cos \phi=\frac{Z_{i}+R-\left(r_{1}+t\right)}{R+r_{1}+t}, \\
& \sin \phi=\frac{r_{1}+\alpha}{R+r_{1}+t} .
\end{aligned}
$$

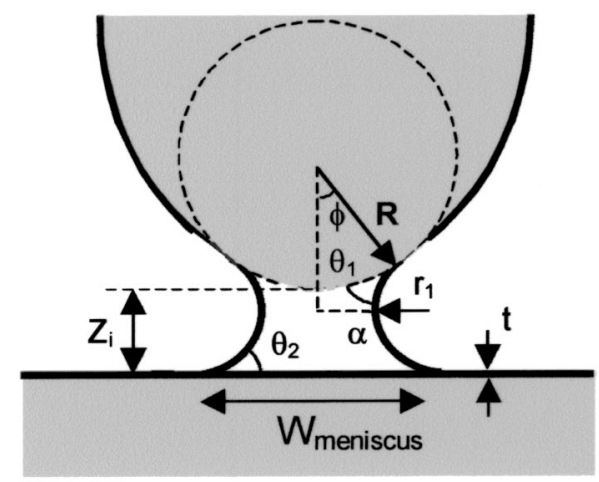

FIG. 2. Schematics of a concave water meniscus formed between a sphere and a flat surface. $\theta_{1}$ and $\theta_{2}$ being the wetting angles both on tip and sample surfaces, $\phi$ the filling angle, $Z_{i}$ tip-sample separation, $R$ the tip radius, $r_{1}$ one of the main curvature radius, $\alpha$ water neck radius and $t$ water thickness adsorbed both on sample and tip surfaces.
Where $\phi$ is the filling angle, $Z_{i}$ is the tip-sample separation, $R$ is the tip radius, $r_{1}$ is one of the main curvature radius, $\alpha$ is the water neck radius (see Fig. 2) and $t$ is water thickness adsorbed both on sample and tip surfaces. ${ }^{27}$

We consider that at the snap-off separation the neck diameter $\alpha$ is zero, then $r_{1}$ is calculated by

$$
\frac{r_{1}^{2}}{\left(R+r_{1}+t\right)^{2}}+\frac{\left[D_{\text {snap-off }}+R-\left(r_{1}+t\right)\right]^{2}}{\left(R+r_{1}+t\right)^{2}}=1,
$$

where $D_{\text {snap-off }}$ is the snap-off separation measured as previously mentioned.

The mean curvature of the meniscus, $\kappa$, is determined by

$$
\kappa=-\frac{\cos \left(\phi+\theta_{1}\right)+\cos \left(\theta_{2}\right)}{R(1-\cos \phi)+Z_{i}-2 t}+\frac{\sin \left(\theta_{1}+\phi\right)}{R \sin \phi},
$$

where $\theta_{1}$ and $\theta_{2}$ are the wetting angles (Fig. 2). We consider that the water perfectly wets both silicon oxide surfaces so $\theta_{1}=\theta_{2}=0$.

If $\kappa$ is constant, then Eq. (3) implies that $r_{1}$ must also be constant. For a known $r_{1}$, the filling angle, $\phi$, can be calculated for any tip-sample separation by Eq. (1). Therefore, it is possible to obtain the meniscus geometry for any tip-sample distance. Also, the bridge diameter $W_{\text {meniscus }}$, is obtained by

$$
W_{\text {meniscus }}=2\left(R+r_{1}+t\right) \sin \phi .
$$

\section{RESULTS AND DISCUSSION}

Figure 3(a) shows the snap-off separation dependence on voltage for three different pulse durations $0.3 \mathrm{~ms}, 30 \mathrm{~ms}$ and 3 s. By using Eqs. (1)-(5) we have calculated the meniscus dimensions from snap-off measurements. The meniscus diameter as a function of applied voltage is shown in Fig. 3(b). An apparently linear dependence of meniscus size on applied voltage is found. Typical rupture distances for the fieldinduced menisci are in the 10-20 nm range. This dependence can be explained as follows. The formation of a water meniscus arises from the interplay of van der Waals forces, condensation energy, surface tension and electrostatic interaction. ${ }^{29}$ Increasing the applied voltage increases the electrostatic energy. So, to minimize the total energy of the system requires the presence of more water molecules in the condensed phase. The liquid meniscus joining tip and sample may be formed by coalescence of water films adsorbed both on tip and sample surfaces, but also from condensation of water molecules from the vapor phase.

Figure 4 shows the snap-off separation and meniscus diameter as a function of pulse duration for a fixed voltage $(24 \mathrm{~V})$. The snap-off separation increases more or less linearly with the logarithm of time. The observed dependence of meniscus dimensions on pulse duration may arise from the observation that condensation is a sequential process. Recent studies about the kinetics of capillary condensation in nanoscale pores show that the time scale over which spontaneously condensed capillaries reach their equilibrium sizes may last thousands of seconds. Kohonen, Maeda, and Christenson ${ }^{30}$ found that an initial water meniscus of $r_{1}=40 \mathrm{~nm}$ is obtained $20 \mathrm{~s}$ after approaching two mica surfaces. However, it takes about $2000 \mathrm{~s}$ to reach the final equilibrium size at $r_{1}$ 

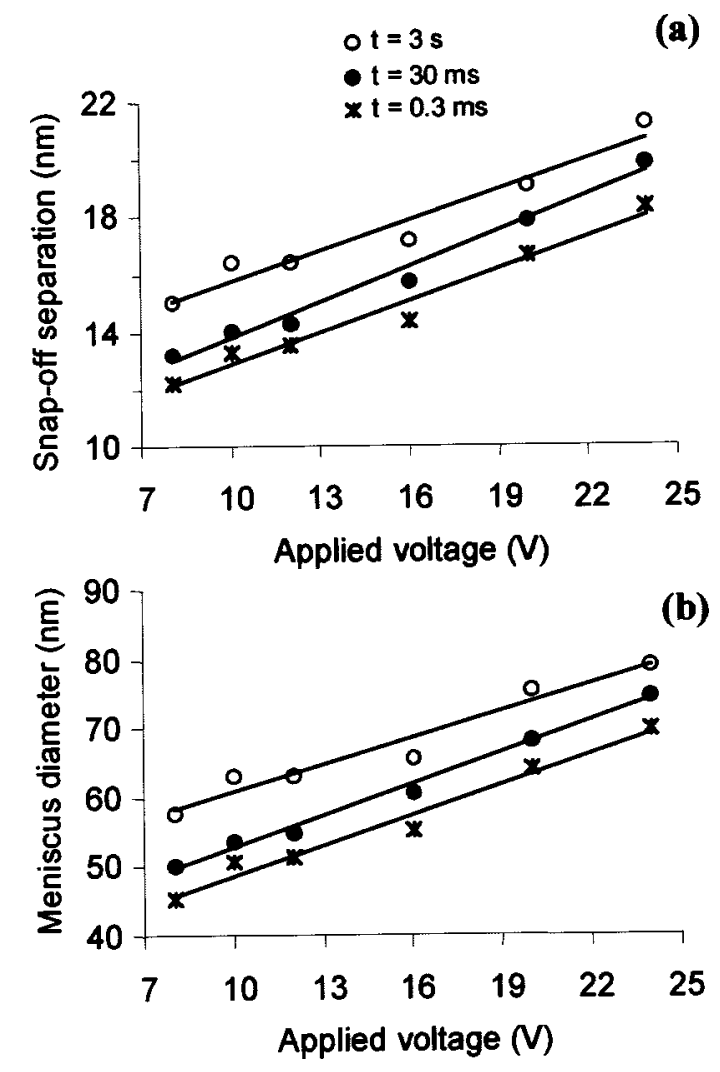

FIG. 3. (a) Snap-off separation dependence on voltage. Lines are drawn as a guide for the eye. (b) Dependence of meniscus diameter on voltage. Data obtained from snap-off separation measurements presented in (a). Experiments were performed at $\mathrm{RH}=45 \%$.

$=100 \mathrm{~nm}$. Those experiments were performed at a relative humidity of 0.9949 . In our case pulse duration values are far smaller than the times reported in the aforementioned experiments. Following the above observations, it seems reasonable to expect that increasing the time that the voltage is applied between tip and surface will allow the condensation of more water layers.

The liquid bridges seem to be stable in a scale of tens of seconds. Several water bridges have been observed for about $50 \mathrm{~s}$. During this time, the oscillation amplitude was smaller than the initial one, showing that the capillary was still present.

The present experiments were performed at relative humidities in the $40-50 \%$ range. For those values, the Kelvin equation $^{31,32}$ predicts a curvature radius ranging from -0.59 to $-0.68 \mathrm{~nm}$. Those values are about one order of magnitude smaller than the ones deduced for field-induced water bridges using Eq. (5). Here we obtain curvature radius values in the range -5 to $-14 \mathrm{~nm}$. According to the Kelvin equation those values should correspond to $90 \%-96 \%$ relative humidities. We attribute those differences to polarization effects involved in the formation of field-induced liquid bridges in the noncontact mode of the AFM. Also, it has been demonstrated that for nanometer-size menisci the Kelvin equation must be modified by the contribution of surface potential and a curvature dependent surface tension in order to obtain a good agreement with molecular dynamics simulations. ${ }^{15}$ The same argument indicates that the Kelvin
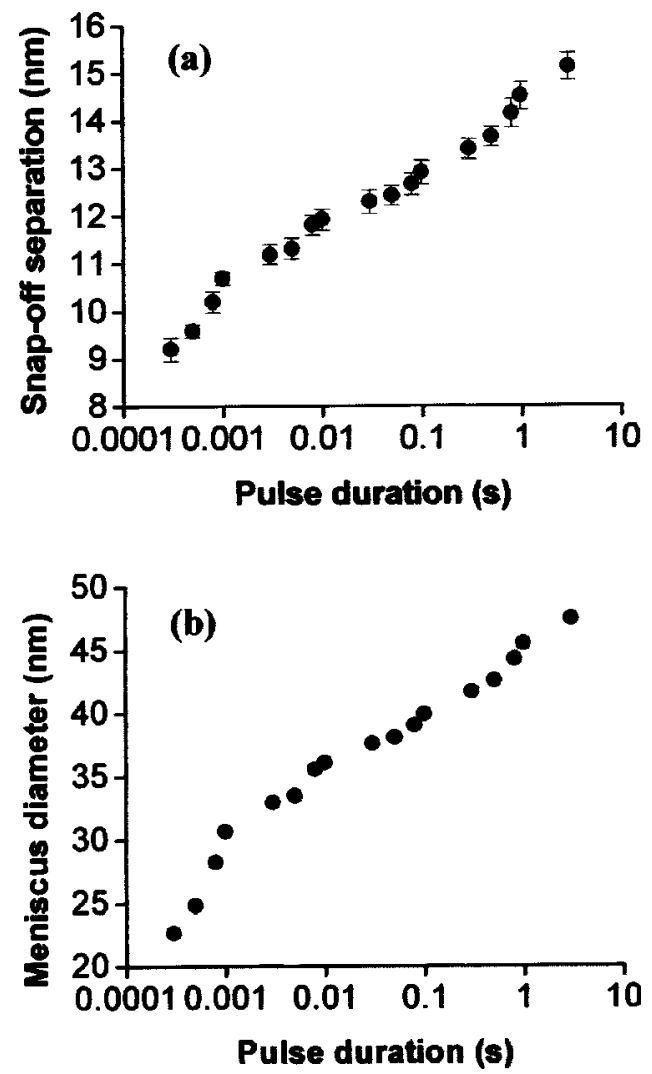

FIG. 4. (a) Snap-off separation dependence on pulse duration. Each point is the average of five different measurements. (b) Dependence of meniscus diameter on pulse duration. Data obtained from snap-off separation measurements presented in (a). The sample is biased negatively with respect to the tip. $\mathrm{RH}=40 \%, V=24 \mathrm{~V}, R=30 \mathrm{~nm}$.

equation should not be used to describe field-induced water bridges.

The controlled manipulation of liquid capillaries is of special relevance in local oxidation lithography, ${ }^{7}$ since the anodic oxidation of the surface is confined within the meniscus diameter. In Fig. 5 we plot the capillary diameter and oxide dot width as a function of the snap-off separation. The data allow comparing capillary and oxide lateral sizes. Oxides and water bridges were obtained for the same voltage

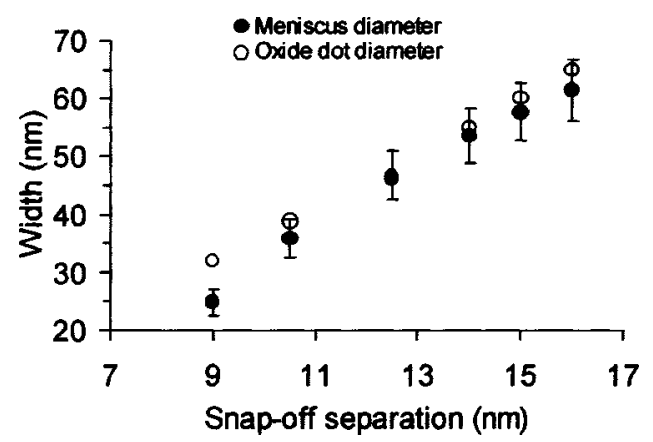

FIG. 5. Dependence of meniscus and oxide dot diameter on snap-off separation. Meniscii diameters are compared with oxide dot diameters as obtained for the same pulse duration and voltage intensity. Error in the determination of meniscus diameter from snap-off separations comes from the error in the determination of tip radius. $V=20 \mathrm{~V}, \mathrm{RH}=45 \%$. Each snap-off separation corresponds to a different pulse duration, $t=0.075,0.15,0.25$, $0.5,1,3 \mathrm{~s}$. 
and pulse duration conditions. ${ }^{33}$ A similar dependence is observed but for short pulses. For pulses below $50 \mathrm{~ms}$, the oxide size is determined almost exclusively by the oxide growth kinetics. As a consequence, for short pulses the oxide size should be smaller than the capillary diameter.

\section{CONCLUSIONS}

We have measured the lateral and vertical dimensions of nanometer-size water bridges. The liquid bridges were formed by the application of an electrical field in an atomic force microscope interface. The measurement of the snap-off separations allows the determination of vertical and lateral size of the capillaries. We have demonstrated that liquid bridges of 5-15 nm curvature radius and $30 \mathrm{~nm}$ in width can be formed and manipulated in a controlled fashion by applying an electrical field between an AFM tip and a surface. The method used to determine the meniscus size is general. It could also be applied to nonfield-induced liquid bridges.

We have studied the dependence of the meniscus size on voltage pulse strength and duration. The liquid bridge size increases by increasing the voltage strength or the pulse duration. The experimental results indicate that the condensation kinetics is highly influenced by the strong electrical fields. The strength of electrostatic interaction controls the meniscus final size.

Finally, the influence that the meniscus width has on the minimum lateral size in local oxidation experiments emphasizes some of the applications of nanometer-size water bridges.

\section{ACKNOWLEDGMENTS}

The authors acknowledge fruitful discussions with S. Gómez-Moñivas and J. J. Sáenz. This work was supported by the Dirección General de Investigación Científica y Técnica (PB98-0471) and the European Commission (MONALISA, GRDI-2000-25592). M. C. acknowledges financial support from the Comunidad Autónoma de Madrid.

${ }^{1}$ E. S. Snow, P. M. Campbell, F. A. Buot, D. Park, C. R. K. Marrian, and R Magno, Appl. Phys. Lett. 72, 3071 (1998).

${ }^{2}$ R. García, M. Calleja, and F. Pérez-Murano, Appl. Phys. Lett. 72, 2295 (1998).

${ }^{3}$ F. S. Chien, C. L. Wu, T. T. Chen, S. Gwo, and W. F. Hsieh, Appl. Phys. Lett. 75, 2429 (1999).

${ }^{4}$ G. Abadal, A. Boisen, Z. J. Davis, O. Hansen, and F. Grey, Appl. Phys. Lett. 74, 3206 (1999).
${ }^{5}$ Y. Gotoh, K. Matsumoto, T. Maeda, E. B. Cooper, S. R. Manalis, H. Fang, S. C. Minne, T. Hunt, H. Dai, J. Harris, and C. F. Quate, J. Vac. Sci. Technol. A 18, 1321 (2000).

${ }^{6}$ M. Calleja and R. García, Appl. Phys. Lett. 76, 3427 (2000).

${ }^{7}$ R. García, M. Calleja, and H. Rohrer, J. Appl. Phys. 86, 1898 (1999).

${ }^{8}$ K. Morimoto, K. Araki, K. Yamashita, K. Morita, and M. Niwa, Appl. Surf. Sci. 117/118, 652 (1997).

${ }^{9}$ M. Tello and R. García, Appl. Phys. Lett. 79, 424 (2001).

${ }^{10}$ A. Avramescu, A. Ueta, K. Uesugi, and I. Suemune, J. Appl. Phys. 88, 3158 (2000)

${ }^{11}$ M. Binggeli and C. M. Mate, Appl. Phys. Lett. 65, 415 (1994).

${ }^{12}$ R. Lipowsky, Curr. Opin. Colloid Interface Sci. 6, 401 (2001).

${ }^{13}$ R. D. Piner, J. Zhu, F. Xu, S. Hong, and C. A. Mirkin, Science 283, 661 (1999).

${ }^{14}$ W. J. Stroud, J. E. Curry, and J. H. Cushman, Langmuir 17, 688 (2001).

${ }^{15}$ M. Miyahara, H. Kanda, T. Yoshioka, and M. Okazaki, Langmuir 16, 4293 (2000).

${ }^{16}$ T. Stifter, O. Marti, and B. Bhushan, Phys. Rev. B 62, 13667 (2000).

${ }^{17}$ A. Valencia, M. Brinkmann, and R. Lipowsky, Langmuir 17, 3390 (2001).

${ }^{18}$ C. Quilliet and B. Berge, Curr. Opin. Colloid Interface Sci. 6, 34 (2001).

${ }^{19}$ L. R. Fisher and J. N. Israelachvili, J. Colloid Interface Sci. 80, 528 (1981).

${ }^{20}$ C. M. Mate, M. R. Lorenz, and V. J. Novotny, J. Chem. Phys. 90, 7550 (1989).

${ }^{21}$ D. L. Malotky and M. K. Chaudhury, Langmuir 17, 7823 (2001).

${ }^{22}$ M. M. Kohonen, and H. K. Christenson, Langmuir 16, 7285 (2000).

${ }^{23}$ L. R. Fisher, R. A. Gamble, and J. Middlehurst, Nature (London) 290, 575 (1981).

${ }^{24}$ M. Schenk, M. Füting, and R. Reichelt, J. Appl. Phys. 84, 4880 (1998).

${ }^{25}$ J. Colchero, A. Storch, M. Luna, J. Gómez-Herrero, and A. M. Baró, Langmuir 14, 2230 (1998).

${ }^{26}$ J. Hu, X. D. Xiao, D. F. Ogletree, and M. Salmeron, Science 268, 267 (1995).

${ }^{27}$ The water thickness, $t$ has been calculated by $t$ $=\left[V A_{123}(t) / 6 \pi \kappa_{B} T \ln \left(p / p_{0}\right)\right]^{1 / 3}$ where $A$ is the Hamaker function and 1-2-3 denotes the solid-liquid-vapor.

${ }^{28}$ F. M. Orr, L. E. Scriven, and A. P. Rivas, J. Fluid Mech. 67, 723 (1975).

${ }^{29}$ J. J. Sáenz and S. Gómez-Moñivas (private communication).

${ }^{30}$ M. M. Kohonen, N. Maeda, and H. K. Christenson, Phys. Rev. Lett. 82, 4667 (1999).

${ }^{31}$ The Kelvin equation has been used to describe the effect of capillary condensation that takes place below saturation by the proximity of two surfaces $r_{k}=\gamma V / k_{B} T \ln \left(p / p_{0}\right)$, where $r_{k}$ is the so-called Kelvin radius which accounts for the curvature of the liquid-vapor interface, $V$ the molar volume, $k_{B}$ is the Boltzmann constant, $T$ is the temperature, $\gamma$ the surface energy of the liquid-vapor interface and $p / p_{0}$ is the relative vapor pressure.

${ }^{32}$ J. Israelachvili, Intermolecular and Surface Forces (Academic, Press London, 1992)

${ }^{33}$ To determine the meniscus size, a negative voltage pulse is applied (with respect to the sample surface). This polarity prevents the formation of a local oxide on the surface. Previously we have verified that the meniscus size is independent of the polarity. 